\title{
Disentangling intrinsic and extrinsic gene expression noise in growing cells
}

\author{
Jie $\operatorname{Lin}^{1}$ and Ariel Amir ${ }^{1}$ \\ ${ }^{1}$ John A. Paulson School of Engineering and Applied Sciences, \\ Harvard University, Cambridge, MA 02138, USA
}

(Dated: August 26, 2020)

\begin{abstract}
Gene expression is a stochastic process. Despite the increase of protein numbers in growing cells, the protein concentrations are often found to be confined within small ranges throughout the cell cycle. Considering the time trajectory of protein concentration as a random walker in the concentration space, an effective restoring force (with a corresponding "spring constant") must exist to prevent the divergence of concentration due to random fluctuations. In this work, we prove that the magnitude of the effective spring constant is directly related to the fraction of intrinsic noise in the total protein concentration noise. We show that one can infer the magnitude of intrinsic, extrinsic, and measurement noises of gene expression solely based on time-resolved data of protein concentration, without any a priori knowledge of the underlying gene expression dynamics. We apply this method to experimental data of single-cell bacterial gene expression. The results allow us to estimate the average protein number and the translation burst parameter.
\end{abstract}

7 Gene expression in all forms of life is subject to noise $8[1-7]$. Experimentally, stochastic gene expression has 9 been intensively studied, mostly in growing cells with ex10 ponentially growing cell volume [8-12] in which the copy 11 numbers of mRNAs and proteins in general double on 12 average during the cell cycle, as widely observed in bac13 terial and eukaryotic cells [8,13-15]. To reduce cell cycle ${ }_{14}$ effects, a more biologically relevant protocol to quantify 15 the stochastic degree of gene expression is to calculate the 16 variability of concentration because most genes in prolif17 erating cells exhibit approximately constant protein con${ }_{18}$ centrations throughout the cell cycle over multiple gen19 erations $[13,16-21]$. In yeast and mammalian cells, most 20 genes also exhibit approximately constant mRNA con21 centrations throughout the cell cycle [14, 22, 23].

${ }_{22}$ Considering the time trajectory of protein concentra23 tion as a one dimensional random walker in the space of 24 concentration, it must be subject to an effective restor25 ing force to prevent the divergence of concentration in 26 the long time limit (note that cell growth contributes to ${ }_{27}$ this restoring force via the effect of dilution, as discussed ${ }_{28}$ extensively in Ref. [19]). However, little is known about ${ }_{29}$ how the strength of this restoring force is related to the 30 stochastic nature of protein concentration. In this work 31 we show that one can in fact infer the contribution of 32 intrinsic and extrinsic noise (which we will define later) 33 to the total gene expression noise from the properties of 34 the restoring force. Previous works on solving this chal35 lenge often rely on particular models of the underlying 36 dynamics of gene expression [24-27]. Here we develop a ${ }_{37}$ novel protocol which is, in contrast, insensitive to many 38 of the details of the gene expression dynamics, and is 39 thus applicable to a broad class of models. The proto${ }_{40} \mathrm{col}$ only relies on analysis of time-series data of protein ${ }_{41}$ concentrations. We expect it to be applicable to expo${ }_{42}$ nentially growing cells such as bacteria, yeast and cancer
43 cells $[8-12]$.

${ }_{44}$ In the following, we first introduce a general framework 45 to study the variability of mRNA and protein concentra46 tions in growing cells. Within the framework, the initi47 ation rates of transcription and translation can be age${ }_{48}$ dependent (here, we define age as the elapsed time since ${ }_{49}$ cell birth), e.g., due to gene dosage effects as well as more 50 complex cell cycle dependencies [15]. We show that inde${ }_{51}$ pendent of the details of the gene expression dynamics, ${ }_{52}$ the variances of mRNA and protein concentrations can 53 always be decomposed into an extrinsic component and ${ }_{54}$ an intrinsic component. In the large cell volume limit, the 55 intrinsic noise vanishes while the extrinsic noise remains ${ }_{56}$ finite [28]. We then introduce our protocol to extract the ${ }_{57}$ fraction of intrinsic noise, extrinsic noise and measure${ }_{58}$ ment noise in the total noise of protein concentrations 59 and finally apply the method to experimental data of 60 bacterial gene expression.

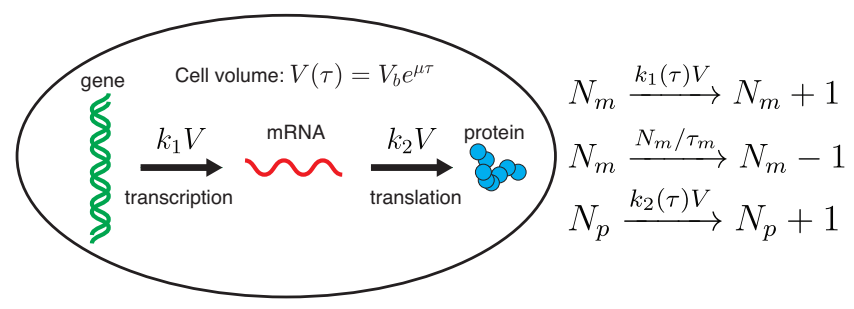

FIG. 1. The cell volume $V$ grows exponentially in time with a growth rate $\mu$ and $\tau$ is the cell age. $k_{1}$ and $k_{2}$ are the transcription rate and translation rate per cell volume which can be age-dependent. The chemical reactions of gene expression are summarized on the right. $N_{m}$ and $N_{p}$ are mRNA and protein numbers respectively. $\tau_{m}$ is the lifetime of mRNA using which one can define the translation burst parameter $\beta \tau_{m}$ (the average number of proteins produced in the lifetime of a single mRNA).

Decomposition of noise.-For simplicity, we consider a ${ }_{64}$ cell growing at a constant growth rate $\mu$ (Fig. 1). When 
${ }_{65}$ the cell divides, the molecules are assumed to be seg- ${ }_{116}$ sic noise in the dual-reporter setup belong to a subset ${ }_{66}$ regated binomially and symmetrically between the two ${ }_{117}$ of those of the extrinsic noise in our framework which ${ }_{67}$ daughter cells [3]. Since for both bacterial and eukary- ${ }_{118}$ includes all possible upstream factors correlated or not 68 otic cells the degradation times of many proteins are ${ }_{119}$ across genes. Therefore, the extrinsic noise from the ${ }_{69}$ longer than the cell cycle duration [29], we consider a ${ }_{120}$ dual-reporter method is typically smaller than the ex70 non-degradable protein. Our results are equally valid for ${ }_{121}$ trinsic noise defined in our current framework, as we will ${ }_{71}$ proteins with a finite degradation rate after some slight ${ }_{122}$ discuss further later.

72 modifications (Supplementary Information, SI A). We al73 low the initiation rates of transcription and translation ${ }_{74}$ per cell volume, $k_{1}, k_{2}$, to be time dependent and, for 75 example, they can exhibit stochastic dynamics. One can 76 further express $k_{2}=\beta m$ where $m$ is the mRNA con77 centration and $\beta$ is the initiation rate of translation per 78 mRNA. Mechanistically $\beta$ is determined by the binding 79 rate of ribosomes to mRNAs and largely determined by so the concentration of ribosomes, which is roughly constant 81 throughout the cell cycle [20].

82 Consider an experiment where one tracks a single lin83 eage of cells over multiple generations, records the data ${ }_{84}$ of protein concentrations $p$ uniformly in time with reso${ }_{85}$ lution $\Delta t$, and finally computes the resulting variance of 86 concentrations based on all collected data. We find that ${ }_{87}$ the resulting variance of protein concentration $\sigma_{p}^{2}$ can be ${ }_{88}$ generally decomposed into three components (SI A):

$$
\sigma_{p}^{2}=\underbrace{\frac{\operatorname{cov}\left(k_{2}, p\right)}{\mu}}_{\text {Upstream noise }}+\underbrace{\left\langle\frac{k_{2}}{2 \mu V}\right\rangle}_{\text {Poisson noise }}+\underbrace{\frac{\overline{p(T)}}{4 \mu V_{b} T}}_{\text {Partitioning noise }}
$$

${ }_{89}$ Here $\operatorname{cov}\left(k_{2}, p\right)=\left\langle k_{2} p\right\rangle-\left\langle k_{2}\right\rangle\langle p\rangle$ and $\langle\cdot \cdot\rangle$ represents aver90 age over time. The first part represents the noise due to ${ }_{91}$ a fluctuating upstream factor, namely, the initiation rate 92 of translation per cell volume. One important source of 93 upstream noise is the fluctuation in mRNA copy num94 ber [28]. The second term represents the noise due to 95 the stochastic production process which we denote as ${ }_{96}$ Poisson noise here. The last term stems from the ran${ }_{97}$ dom partitioning during cell division where $T=\ln 2 / \mu$ 98 is the doubling time. The Poisson noise and the parti99 tioning noise scale with the inverse of cell volume and 100 their contributions to the variance $\sigma_{p}^{2}$ become negligible 101 in the large cell volume limit conditioned on a fixed aver102 age concentration. In contrast, the upstream noise stems 103 from the fluctuation in the translation rate per cell vol104 ume and it does not vanish in the large cell volume limit.

(a) Computing the protein production rate

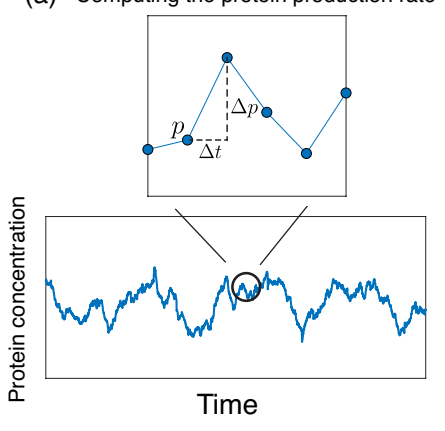

(b) Without measurement noise

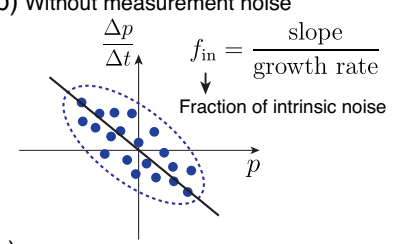

(c) With measurement noise

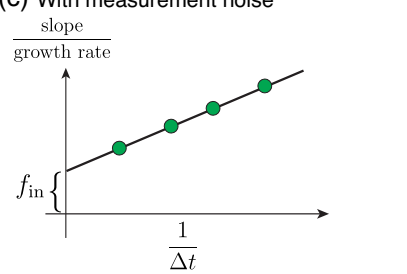

FIG. 2. (a) Given a time series of protein concentration, we first compute the protein production rate as the discrete time derivative of protein concentration with a time interval $\Delta t$. (b) Next, we perform a linear fit of the protein production rate $\Delta p / \Delta t$ against the current protein concentration $p$ and consider the absolute value of the fitted slope. In the case of negligible measurement noise, the fraction of intrinsic noise is the ratio between the slope and growth rate. (c) For experimental data with measurement noise, we compute the protein production rate for multiple time intervals $\Delta t$ and repeat the protocol in (b) for each time interval. Finally, we perform a linear fit of the normalized slopes against $1 / \Delta t$ and infer the fraction of intrinsic noise from the intercept.

\section{4}

125 Extracting the fraction of intrinsic and extrinsic 126 noise.-In the following, we discuss a protocol to dis127 entangle the contribution of intrinsic and extrinsic noise 128 to the total noise based on the time trajectory of con129 centration (Fig. 2a, b). We consider a discrete incre130 ment of protein concentration over a small time window, ${ }_{131} \Delta p(t)=p(t+\Delta t)-p(t)$, which can be expressed as

$$
\Delta p(t)=\frac{\Delta N_{p}(t)}{V(t)}-\mu p(t) \Delta t
$$

${ }_{105}$ We therefore define the sum of the Poisson noise and the ${ }_{132}$ where $\Delta N_{p}(t)$ is a random variable from a Poisson dis106 partitioning noise as intrinsic and the upstream noise as ${ }_{133}$ tribution with mean $k_{2}(t) V \Delta t$ assumed constant within 107 extrinsic, consistent with previous works $[28,30]$. We nu- ${ }_{134}$ the small time interval $\Delta t$. The second term on the right 108 merically confirm the validity of the noise decomposition 135 side arises from dilution due to cell growth. The pro109 for multiple gene expression dynamics including stochas- 136 tein concentration fluctuates but does not diverge in the 110 tic transcription and translation rate (SI B, Fig. S1). $\quad{ }_{137}$ long time limit, therefore we can make an analogy with a ${ }_{111}$ We remark that the definition of extrinsic noise in our ${ }_{138}$ Brownian particle attracted to a fixed point with a linear 112 framework is different from the extrinsic noise inferred ${ }_{139}$ restoring force equal to $-k x$ where $k$ is the spring con113 from the dual-reporter setup [1,31], which is defined 140 stant and $x$ is the particle position relative to its equi114 as the correlated noise of two identical genes controlled ${ }_{141}$ librium point. In the case of a Brownian particle, one 115 by the same promoters. The possible sources of extrin- ${ }_{142}$ can find the spring constant of the restoring force as the 
(a) Constitutively expressed gene
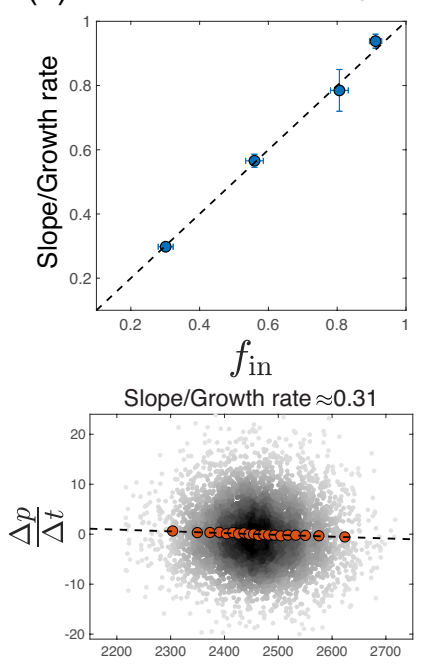

$p$ (b) Transcriptional bursting gene
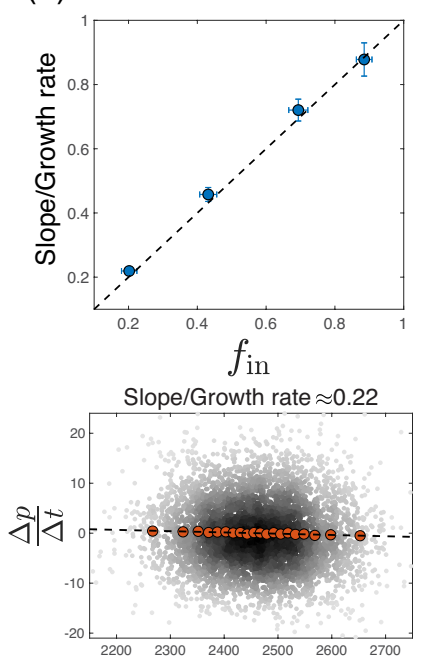

$p$

FIG. 3. (a) Simulation of a constitutively expressed gene. (Upper) We compare the predicted fraction of intrinsic noise ( $y$ axis) to the measured value ( $x$ axis). (Bottom) An example of the raw numerical data with the binned data shown as well (red circles). The dashed line is the linear fit of the raw data. The same analysis also applies to panel (b). Here $k_{1}=10$. (b) Simulation of a transcriptional bursting gene with $k_{\text {on }}=$ $10, k_{\text {off }}=10, k_{1}=20$. In all upper panels, the doubling time $T=60, \tau_{m}=10$, and $\beta$ is varied so that $\log _{10} \beta=$ $-2,-1.5,-1,-0.5$. In all bottom panels, $\log _{10} \beta=-0.5$. We compute the time-derivative of protein concentration with a time interval $\Delta t=0.5$. The errorbars are computed as the standard deviation of 5 independent simulations.

${ }_{143}$ slope in the linear fitting of the discrete velocity $\Delta x / \Delta t$ 144 vs. $x$. In the case of protein concentration, one can do 145 a similar analysis by linearly fitting the protein produc${ }_{146}$ tion rate $\Delta p / \Delta t$ vs. $p$. Considering a least square linear ${ }_{147}$ fitting, the slope of the linear fitting is found to be

$$
S \equiv-\frac{\operatorname{cov}\left(\frac{\Delta p(t)}{\Delta t}, p(t)\right)}{\sigma_{p}^{2}}=\mu-\frac{\operatorname{cov}\left(k_{2}(t), p(t)\right)}{\sigma_{p}^{2}} .
$$

${ }_{148}$ where we have used Eq. 2. If the covariance between 149 the translation rate and protein concentration vanishes, 150 the spring constant of the restoring force is simply the 151 growth rate. Combined with Eq. 1, we find that the slope 152 is proportional to the growth rate and the proportional ${ }_{153}$ constant is precisely the fraction of intrinsic noise in the 154 total protein concentration noise variance:

$$
S=\mu\left(1-\frac{\operatorname{cov}\left(k_{2}(t), p(t)\right)}{\mu \sigma_{p}^{2}}\right)=\mu f_{\text {in }} .
$$

${ }_{155}$ The above equation shows that we can extract the frac156 tion of intrinsic noise $f_{\text {in }}$ in the total noise simply by ${ }_{157}$ linearly fitting the time derivative of the protein concen${ }_{158}$ tration against the current protein concentration without 159 any a priori knowledge of the underlying gene expression

${ }_{160}$ dynamics. Extrinsic noise reduces the slope in the lin${ }_{161}$ ear fitting which precisely equals the growth rate $\mu$ in 162 the absence of extrinsic noise. An extended discussion 163 along with an intuitive argument on the effects of extrin164 sic noise based on a Langevin equation is provided in SI ${ }_{165} \mathrm{~F}$. We remark that our protocols are also applicable to 166 nongrowing cells with a constant cell volume given the 167 lifetime of the studied protein is known (SI A).

${ }_{168}$ Analysis of synthetic data.-We test Eq. 4 on syn169 thetic data, first considering a constitutively expressed 170 gene where the initiation rate of transcription per cell 171 volume $k_{1}$ is constant as is the initiation rate of transla172 tion per mRNA $\beta$. This assumption corresponds to the 173 case in which both RNA polymerase and ribosomes are 174 limiting for gene expression, as discussed in detail in Ref. $175[19]$. We compute $f_{\text {in }}$ numerically using Eq. 1 and com176 pare it with the prediction from Eq. 4, finding excellent 177 agreement (Fig. 3a). To test the robustness of our proto${ }_{178} \mathrm{col}$, we also verify our theoretical results on various other 179 gene expression dynamics: (1) the scenario of transcrip180 tional bursting where a gene switches from "off" state to ${ }_{181}$ "on" state with rate $k_{\text {on }}$ and vice versa with rate $k_{\text {off }}$ (Fig. $1823 \mathrm{~b})$; (2) a gene with a constant transcription rate propor183 tional to the gene number which doubles in the middle of 184 the cell cycle (Fig. S2a); this scenario corresponds to the 185 situation when the gene copy number is the sole limiting 186 factor of transcription [19]; (3) a gene with a transcrip${ }_{187}$ tion rate modulated throughout the cell cycle due to a fi${ }_{188}$ nite period of DNA replication (Fig. S2b, see details in SI ${ }_{189} \mathrm{E}$ ); (4) a gene with a fluctuating transcription rate (Fig. $190 \mathrm{~S} 2 \mathrm{c})$; (5) a gene with a fluctuating translation rate per 191 mRNA (Fig. S2d). In all cases, the predicted fractions 192 of intrinsic noise match the actual values well. We also 193 find that in all cases increasing the translation rate per 194 mRNA $\beta$ increases the fraction of extrinsic noise as the 195 effects of upstream noise are amplified, consistent with 196 the analytical results of constitutively expressed genes 198 (SI C, D).

199 In our framework the extrinsic noise is extracted from 200 the time trajectory of the protein concentration of a sin201 gle gene, which is distinct from that of the dual-reporter 202 method. If the two genes in the dual-reporter setup share 203 the same fluctuating translation rate $k_{2}(t)$, the two def204 initions of extrinsic noise will coincide (SI G, Fig. S5a). 205 However, if the correlated noise between the two genes 200 is at the transcriptional level, the extrinsic noise inferred ${ }_{207}$ from the dual-reporter will be smaller than the one ex208 tracted from our protocol, which we confirm numerically 209 (Fig. S5b).

${ }_{210}$ Analysis of experimental data.-Experimentally, the ${ }_{211}$ measured protein concentration is always augmented by 212 measurement noise. To model the effects of measurement ${ }_{213}$ noise, we assume the measured protein concentration at 214 time $t$ to equal

$$
p(t)=p_{0}(t)+\eta(t)
$$



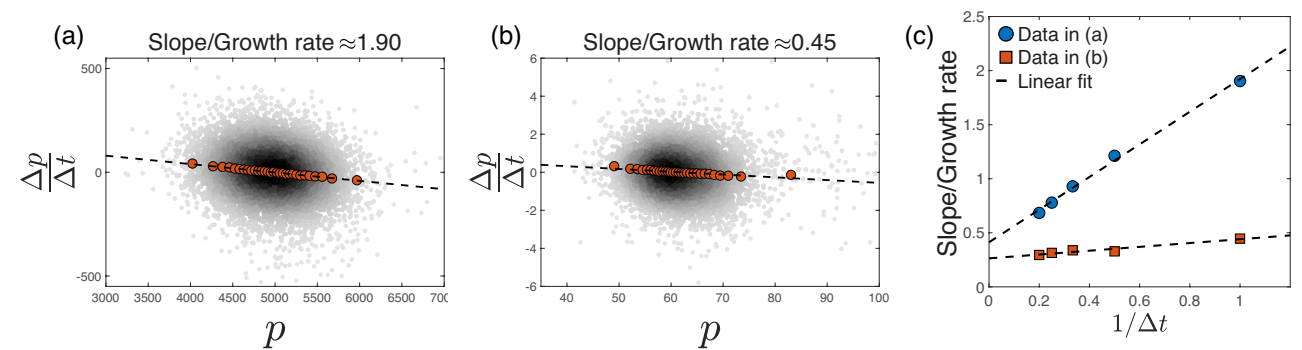

(d)

FIG. 4. (a) We compute the time derivative of protein concentration as a function of the current protein concentration using data from Ref. [32] and the measured slope normalized by the growth rate is 1.90. The time interval used is $\Delta t=1$ min and the growth rate is $\mu=0.0213 \mathrm{~min}^{-1}$. (b) We repeat the analysis using another data from Ref. [8] where the measured slope normalized by the growth rate is 0.45 . Here $\Delta t=1 \mathrm{~min}$ and $\mu=0.0327 \mathrm{~min}^{-1}$. (c) We adjust the time interval to compute the time derivative of protein concentration and compute the slope in the linear fit of $\Delta p / \Delta t v s$. $p$. The normalized slope is linearly fitted as a function of the inverse of the time interval. The fraction of intrinsic noise in the total noise can be calculated from the intercept of the linear fit. We also infer the fraction of measurement noise in the total noise from the slope of the linear fit. (d) We summarize the calculated fractions of different noise for the two data sets. $f_{\text {in }}$ : the fraction of intrinsic noise. $f_{\mathrm{ex}}$ : the fraction of extrinsic noise. $f_{\mathrm{me}}$ : the fraction of measurement noise.

${ }_{215}$ where $p_{0}(t)$ is the actual protein concentration and $\eta(t){ }_{249}$ slope. The results are summarized in Fig. $4 \mathrm{D}$. To justify 216 is the measurement noise term assumed uncorrelated be- 250 the assumption of uncorrelated measurement noise, we ${ }_{217}$ tween different measurements. We will revisit this as- ${ }^{251}$ show that the scaling with $\Delta t$ in Eq. 6 is violated for 218 sumption later on and show that the datasets we an- 252 correlated measurement noise (SI H, Fig. S6).

219 alyzed are consistent with it. The covariance between ${ }_{253}$ In this way we find that the ratio between the mea${ }_{220} \Delta p / \Delta t$ and $p$ becomes $\operatorname{cov}\left(\frac{\Delta p}{\Delta t}, p\right)=\operatorname{cov}\left(\frac{\Delta p_{0}}{\Delta t}, p_{0}\right)-{ }_{254}$ surement noise and the total noise in the two data sets ${ }_{221} \sigma_{\eta}^{2} / \Delta t$. Compared with Eq. 4, the slope in the linear ${ }_{255}$ are respectively $17 \%$ and $10 \%$ in terms of their standard 222 fitting of $\Delta p / \Delta t$ vs. $p$ is modified to

$$
S \equiv-\frac{\operatorname{cov}\left(\frac{\Delta p}{\Delta t}, p\right)}{\sigma_{p}^{2}}=\mu\left(f_{\mathrm{in}}+\frac{\sigma_{\eta}^{2}}{\mu \sigma_{p}^{2} \Delta t}\right) .
$$
256 deviations. We can further use our analytic results for 257 constitutively expressed genes as used in these experi-

(6) 258 ments to estimate the average copy numbers of proteins 259 at cell birth and the translation burst parameter $\beta \tau_{m}$ (see ${ }_{223}$ We confirm Eq. 6 using numerical simulations with arti- 260 Eqs. S28, S29 in SI C) [30]. We find that $N_{p} \approx 230$ at cell ${ }_{224}$ ficial measurement noise. In this case since $\sigma_{\eta}^{2}$ is assigned ${ }_{261}$ birth, $\beta \tau_{m} \approx 1.37$ for Data in Fig. 4(a), and $N_{p} \approx 210$ 225 and $f_{\text {in }}$ is known, we can directly compare the left and 262 at cell birth, $\beta \tau_{m} \approx 2.81$ for Data in Fig. 4(b).

226 right sides of Eq. 6, obtaining good agreement (SI H, 263 Summary and outlook.- - In this work, we start from a ${ }_{227}$ Fig. S6). Experimentally, the fluorescence level may not 264 general framework of stochastic gene expression in expo${ }_{228}$ accurately reflect the instantaneous protein number due 265 nentially growing cells. Our approach allows us to take 229 to a finite maturation time of the fluorescent protein. 266 into account the cell growth and division explicitly and ${ }_{230}$ We have confirmed that the effects of a finite matura- 267 study the variability in protein concentrations, directly ${ }_{231}$ tion time does not affect our results for experimentally ${ }_{268}$ relevant to experiments on proliferating cells such as bac${ }_{232}$ relevant values of the maturation times [33] (SI I, Fig. 269 teria, yeast or cancer cells. We derive a broadly appli$233 \mathrm{~S} 7$ ).

270 cable decomposition of the protein concentration noise, ${ }_{234}$ We analyze two datasets of $E$. coli growth. In both, ${ }_{271}$ finding that the total noise can be expressed as the sum ${ }_{235}$ cells are exponentially growing and a fluorescent protein 272 of the noise due to upstream factors, the Poisson noise ${ }_{236}$ is constitutively expressed $[8,32]$. A single lineage of 273 due to the random process of production and degrada${ }_{237}$ cells is tracked for about 100 generations with cell vol- 274 tion, and the noise due to random partitioning during cell 238 ume and fluorescence level measured simultaneously. In ${ }_{275}$ division. These results are independent of the underlying 239 both cases, the time interval between two consecutive ${ }_{276}$ details of the particular dynamics of mRNA and protein 240 data points is $1 \mathrm{~min}$. To compute $f_{\text {in }}$ for the experimen- 277 synthesis. Given a time trajectory of protein concentra${ }_{241}$ tal data, we increase the time interval to compute $\Delta p / \Delta t{ }_{278}$ tion, one may linearly fit the protein production rate as 242 and find the slopes in the linear fitting of $\Delta p / \Delta t$ vs. $p 279$ a function of the protein concentration. We find that the ${ }_{243}$ for each time interval (see examples for $\Delta t=1 \mathrm{~min}$ in 280 slope of the fit, normalized by the growth rate, precisely ${ }_{244}$ Fig. 4A, B). We then linearly fit the resulting slopes as ${ }_{281}$ equals the fraction of intrinsic noise in the total protein 245 a function of $1 / \Delta t$ (Fig. $2 \mathrm{C}$ ) and the results agree well 282 concentration noise in the absence of measurement noise. 246 with the prediction of Eq. 6 (Fig. 4C). Notably, this ${ }_{283}$ We verify our theoretical framework on synthetic data of ${ }_{247}$ allows us to infer both $f_{\text {in }}$ as the intercept of the lin- ${ }_{284}$ protein concentrations both for constitutively expressed ${ }_{248}$ ear fit, and the fraction of measurement noise from the 285 genes and genes with various underlying gene expression 
286 dynamics.

${ }_{287}$ Importantly, we generalize our protocol to analyze ex- 340 ${ }_{288}$ perimental data of E. coli gene expression and show how ${ }^{341}$ 289 a generalization of the method can simultaneously reveal 290 the fraction of measurement noise in addition to that 291 of intrinsic and extrinsic noise. Our framework predicts 292 that the slope in the linear fitting of the time deriva293 tive of protein concentration vs. the current protein con${ }_{294}$ centration has a linear dependence on the inverse of the 295 time-interval used in the experiments, which agrees well 296 with the experimental results. Assuming a model of a ${ }_{297}$ constitutively expressed protein as used in these experi298 ments, our approach also allows us to infer the average 299 copy numbers of proteins at cell birth as well as the trans300 lation burst parameter, relying only on time-series data 301 of protein concentrations in proliferating cells.

${ }_{302}$ The generality of our approach and the agreement be303 tween experiments and theoretical predictions suggests 304 that the method should be broadly applicable and will 305 serve as a useful tool for gene expression analysis. Our 306 protocol to extract the intrinsic and extrinsic noise re307 lies only on the time trajectory of protein concentration 308 of a single gene, in contrast to the dual-reporter proto$309 \mathrm{col}$ which relies on measuring protein concentrations of 310 two identical genes. Combing our method with the dual311 reporter method, one can further decompose the extrin312 sic noise into correlated and uncorrelated components. 313 Theoretically, our work elucidates how various processes ${ }_{314}$ contribute to the gene expression noise in proliferating 315 cells, and paves the way to further studies on the nature 316 of the widely-observed yet poorly understood extrinsic 317 noise in gene expression.

318 We thank Ido Golding and Lydia Robert for useful dis319 cussions and feedback. A.A. was supported by NSF CA320 REER grant 1752024 and the Harvard Dean's Competi321 tive Fund. A.A. and J.L. thank support from Harvard's 322 MRSEC (DMR-1420570). 344 346
347 $348[1$ 350 351 353 354
355 355
356 356 357 $358[1$ 359 $360[1$
361 361 363 364 366 367 368
369 $369 \quad[20$ 370
371 371
372

[1] M. B. Elowitz, A. J. Levine, E. D. Siggia, and P. S. ${ }^{385}$ Swain, Science 297, 1183 (2002).

[2] J. M. Raser and E. K. O'shea, Science 304, 1811 (2004).

[3] I. Golding, J. Paulsson, S. M. Zawilski, and E. C. Cox, Cell 123, 1025 (2005).

[4] J. R. Newman, S. Ghaemmaghami, J. Ihmels, D. K. Breslow, M. Noble, J. L. DeRisi, and J. S. Weissman, Nature 441, 840 (2006).

[5] A. Raj and A. van Oudenaarden, Cell 135, 216 (2008).

[6] Y. Taniguchi, P. J. Choi, G.-W. Li, H. Chen, M. Babu, J. Hearn, A. Emili, and X. S. Xie, Science 329, 533 (2010).

[7] N. Eling, M. D. Morgan, and J. C. Marioni, Nature Reviews Genetics 20, 536 (2019).

[8] P. Wang, L. Robert, J. Pelletier, W. L. Dang, F. Taddei, A. Wright, and S. Jun, Current Biology 20, 1099 (2010). 342 343 345 349 352 $365[18$

[9] M. Godin, F. F. Delgado, S. Son, W. H. Grover, A. K. Bryan, A. Tzur, P. Jorgensen, K. Payer, A. D. Grossman, M. W. Kirschner, et al., Nature Methods 7, 387 (2010).

10] M. Campos, I. V. Surovtsev, S. Kato, A. Paintdakhi, B. Beltran, S. E. Ebmeier, and C. Jacobs-Wagner, Cell 159, 1433 (2014).

11] S. Taheri-Araghi, S. Bradde, J. T. Sauls, N. S. Hill, P. A. Levin, J. Paulsson, M. Vergassola, and S. Jun, Current Biology 25, 385 (2015).

12] N. Cermak, S. Olcum, F. F. Delgado, S. C. Wasserman, K. R. Payer, M. A Murakami, S. M. Knudsen, R. J. Kimmerling, M. M. Stevens, Y. Kikuchi, A. Sandikci, M. Ogawa, V. Agache, F. Baleras, D. M. Weinstock, and S. R. Manalis, Nature Biotechnology 34, 1052 (2016).

13] H. A. Crissman and J. A. Steinkamp, The Journal of Cell Biology 59, 766 (1973).

14] O. Padovan-Merhar, G. P. Nair, A. G. Biaesch, A. Mayer, S. Scarfone, S. W. Foley, A. R. Wu, L. S. Churchman, A. Singh, and A. Raj, Molecular Cell 58, 339 (2015).

15] M. Wang, J. Zhang, H. Xu, and I. Golding, Nature microbiology 4, 2118 (2019).

16] S. Elliott and C. McLaughlin, Proceedings of the National Academy of Sciences 75, 4384 (1978).

17] N. Brenner, E. Braun, A. Yoney, L. Susman, J. Rotella, and H. Salman, The European Physical Journal E 38, 1 (2015).

18] N. Walker, P. Nghe, and S. J. Tans, BMC Biology 14, 11 (2016).

19] J. Lin and A. Amir, Nature Communications 9, 4496 (2018).

20] G. E. Neurohr, R. L. Terry, J. Lengefeld, M. Bonney, G. P. Brittingham, F. Moretto, T. P. Miettinen, L. P. Vaites, L. M. Soares, J. A. Paulo, et al., Cell 176, 1083 (2019).

21] N. Nordholt, J. H. van Heerden, and F. J. Bruggeman, Current Biology 30, 2238 (2020).

22] H. Kempe, A. Schwabe, F. Crémazy, P. J. Verschure, and F. J. Bruggeman, Molecular Biology of the Cell 26, 797 (2015).

23] X.-M. Sun, A. Bowman, M. Priestman, F. Bertaux, A. Martinez-Segura, W. Tang, C. Whilding, D. Dormann, V. Shahrezaei, and S. Marguerat, Current Biology 30, 1217 (2020).

[24] D. L. Jones, R. C. Brewster, and R. Phillips, Science 346, 1533 (2014).

[25] R. D. Dar, B. S. Razooky, L. S. Weinberger, C. D. Cox, and M. L. Simpson, PLoS One 10, 1 (2015).

[26] O. Lenive, P. D. Kirk, and M. P. Stumpf, BMC Systems Biology 10, 81 (2016).

[27] M. Soltani, C. A. Vargas-Garcia, D. Antunes, and A. Singh, PLoS Comput Biol 12, e1004972 (2016).

[28] J. Paulsson, Nature 427, 415 (2004).

[29] R. Milo and R. Phillips, Cell biology by the numbers (Garland Science, 2015).

393 [30] J. Paulsson, Physics of Life Reviews 2, 157 (2005).

394 [31] P. S. Swain, M. B. Elowitz, and E. D. Siggia, Proceedings of the National Academy of Sciences 99, 12795 (2002).

96 [32] Y. Tanouchi, A. Pai, H. Park, S. Huang, N. E. Buchler, and L. You, Scientific Data 4, 170036 (2017).

998 [33] E. Balleza, J. M. Kim, and P. Cluzel, Nature Methods 15, 47 (2018). 